in vivo $36: 473-481(2022)$

doi:10.21873/invivo.12727

\title{
Combination of GATA3, SOX-10, and PAX8 in a Comprehensive Panel to Diagnose Breast Cancer Metastases
}

\author{
KIYONG NA ${ }^{1}$, HA YOUNG WOO ${ }^{2}$, SUNG-IM DO ${ }^{3}$ and SO-WOON KIM ${ }^{1}$ \\ ${ }^{1}$ Department of Pathology, Kyung Hee University Hospital, \\ Kyung Hee University College of Medicine, Seoul, Republic of Korea; \\ ${ }^{2}$ Department of Pathology, National Cancer Center, Goyang, Republic of Korea; \\ ${ }^{3}$ Department of Pathology, Kangbuk Samsung Hospital, \\ Sungkyunkwan University School of Medicine, Seoul, Republic of Korea
}

\begin{abstract}
Background/Aim: It can be difficult to establish the origin of a tumor in metastatic breast cancer $(M B C)$, especially with triple-negative breast cancer (TNBC) or high-grade features. We evaluated the diagnostic utility of GATA3, SOX10, and PAX8 panels in MBC by comparing their expression in each molecular subtype of $M B C$. Patients and Methods: We evaluated $84 \mathrm{MBC}$ and 37 primary TNBC cases using GATA3, SOX10, and PAX8 staining in whole tissue sections. Results: GATA3 was least sensitive in the detection of metastatic TNBC (metastatic non-TNBC, 0.95; metastatic TNBC, 0.37). SOX10 had the lowest overall sensitivity (0.12) but was elevated in metastatic TNBC, even higher than GATA3 (0.59 vs. 0.37). The combination of GATA3, SOX10, and PAX8 expression showed the highest detection rate $(M B C, 0.94$; metastatic non-TNBC, 0.95; metastatic TNBC, 0.93). Conclusion: We recommend combining GATA3, SOX10, PAX8 expression profiling to confirm breast as the site of origin in metastatic $M B C$.
\end{abstract}

This article is freely accessible online.

Correspondence to: So-Woon Kim, Department of Pathology, Kyung Hee University Hospital, Kyung Hee University College of Medicine, 26 Kyungheedae-ro, Dongdaemun-gu, Seoul 02447, Republic of Korea. Tel: +82 29588740, Fax: +82 29588744, e-mail: sowoonkim86@gmail.com and Sung-Im Do, Department of Pathology, Kangbuk Samsung Hospital, Sungkyunkwan University School of Medicine, 29, Saemunan-ro, Jongno-gu, Seoul 03181, Republic of Korea. Tel: +82 220012389, Fax: +82 220012398, e-mail: sungim.do@samsung.com

Key Words: GATA3, SOX10, PAX8, breast cancer, triple-negative breast cancer.
As breast cancer metastasis is prevalent and metastatic lesions often need a surgical biopsy, challenges surrounding these is commonly encountered in clinical practice (1). Organ-specific markers are important for the histopathologic detection of metastatic breast carcinoma (MBC) in abnormal locations or clinically unsuspected primary cancers (2). A tissue biopsy can confirm the metastasis of a suspected lesion, and it is used to investigate changes in the molecular subtype of breast cancer after metastasis (1). In most cases, breast cancer metastasis is strongly supported by i) a history of primary breast cancer (PBC); ii) morphologically similar metastasis to the previous $\mathrm{PBC}$ determined through a histological slide review; and iii) stable expression of both breast lineage markers and biomarkers, such as estrogen receptor (ER) (3). While supporting the mammary gland origin of ER-positive breast cancer is relatively straightforward, histopathological diagnosis of metastatic breast cancer (MBC) is difficult for ER-negative or triple-negative breast cancer (TNBC). This is due to complex or incomplete clinical information, which often coexists other ERexpressing tumors (e.g., high-grade serous carcinoma of the ovary) (4).

No immunohistochemical staining (IHC) marker is completely specific for breast cancer, and the ER status of the carcinoma affects the IHC sensitivity (4). The sensitivity of standard breast lineage markers, such as mammaglobin (MMG) and gross cystic disease fluid protein 15 (GCDFP-15), depend on the tumor subtype, with the lowest sensitivity in grade 3, ER-negative, or TNBC cases $(5,6)$. Recently, GATA-binding protein 3 (GATA3) has emerged as a highly sensitive marker for breast cancer with an expression rate $>90 \%$ (7). However, despite a higher sensitivity for ER-positive breast cancer, its sensitivity towards TNBC is low, often $<40 \%$ (8). 
Another promising marker found in $40 \%-70 \%$ of TNBC is Sry-related HMG box (SOX)10, a transcription factor protein belonging to the SOX family (9-11). SOX10 has been identified even in TNBCs that are negative for GATA3 (11), and can, therefore, complement other breast markers in determining the breast origin of metastatic carcinoma.

Of note, encountering high-grade MBC with micropapillary features of the axilla requires careful consideration. In such a situation, differential diagnosis of high-grade MBC and high-grade serous ovarian carcinoma, based on the ER status and histological features, is difficult (12). Several IHC markers are used for diagnosis to establish the origin of carcinomas, such as paired box 8 (PAX8) and WT-1, but none of them are completely specific for ovarian or breast cancers $(4,13)$. PAX8 shows a higher specificity than WT-1 in differentiating between high-grade ovarian and breast carcinomas, but the specificity varies depending on the antibody clone used $(4,13,14)$. Therefore, it is critical to include markers of both mammary origin, such as GATA3 or GCDFP, as well as gynecological tract origin, such as PAX8, in the differential diagnosis of a micropapillary carcinoma in the breast or axilla (4).

In this study, we analyzed the expression levels of GATA3, SOX10, and PAX8 in $84 \mathrm{MBC}$ and 37 primary TNBC (p-TNBC) samples by studying their histological and molecular features. We also determined the most suitable marker combination panels for detecting MBC, based on the molecular type of the tumor.

\section{Patients and Methods}

Patient selection and tissue samples. This study (2020-03-037) was approved by the Institutional Review Board of Kyung Hee University Hospital (Seoul, Republic of Korea). We searched for all cases of MBC diagnosed between January 1995 and January 2019 in the pathology database of the Kyung Hee University Hospital. We collected formalin-fixed, paraffin-embedded specimens from metastatic sites with matched primary resected specimens. Excluding cases with regional lymph node metastasis only, we enrolled 84 patients with distant metastasis. MBCs were diagnosed in 62 of 84 patients $(78 \%)$ using small biopsy specimens, and in the remaining 22 patients (22\%) using metastasectomy specimens. For an in-depth analysis, we included 37 cases of p-TNBC in the casecohort, out of whom none had a history of other malignancies. The PBCs in all the 37 cases were surgically resected.

Review of pathological diagnosis. The tumors were histologically classified according to the World Health Organization System (12). Two experienced pathologists ( $\mathrm{KN}$ and $\mathrm{SWK}$ ) reviewed all the available hematoxylin-eosin slides of the $84 \mathrm{MBCs}$ and $37 \mathrm{PBCs}$ through routine microscopic examination and selected the most representative slide from each case for the IHC staining. Two cases with inter-observer discrepancy were re-classified after obtaining a third opinion (HSK). The histopathological review focused on the histological subtype and grade, i) including predominant growth patterns, ii) nuclear features, and iii) mitotic activity, based on the Nottingham histological score system (13). We collected histological subtypes and tumor stages for all the external cases but were unable to retrieve their histological grades and molecular subtypes. Clinical data, including patient demographics and procedures performed in these patients, were collected from electronic medical records and reviewed.

Immunohistochemistry and fluorescence in situ hybridization. Whole tissue sections of PBC and MBC from the most representative tumor blocks were obtained and stained with breast cancer markers. Before performing an immunohistochemistry study for GATA3, SOX10, and PAX8, all previously used IHC markers were reviewed. ER, progesterone receptor (PR), human epidermal growth factor receptor 2 (HER2), and Ki-67 labeling index, along with GATA3, SOX10, and PAX8 immunostaining, were used for the molecular subtyping of breast cancer, using an automatic instrument (Vision Biosystems, Mount Waverly, Victoria, Australia) according to the manufacturer's recommendations. We performed an antigen retrieval procedure using Bond ER2 solution for $30 \mathrm{~min}$ at $100^{\circ} \mathrm{C}$. Endogenous peroxidases were quenched by incubating with hydrogen peroxide for $5 \mathrm{~min}$. The sections were incubated for $15 \mathrm{~min}$ at ambient temperature with primary antibodies against GATA3 (1:300, clone L50-823, Ventana Medical Systems SA, Strasbourg, France), SOX10 (1:200, clone EP268, Ventana Medical Systems SA), PAX8 (1:50, clone MRQ-50, Cell Marque, Rocklin, CA, USA), ER (1:200, clone 6F11, Leica Biosystems, Buffalo Grove, IL, USA), PR (1:200, clone 16, Leica Biosystems), HER2 (1:1, clone 4B5, Ventana Medical Systems SA), and Ki-67 (1:100, clone MIB1, Dako, Carpinteria, CA, USA). Nuclei were counterstained with hematoxylin. We concurrently stained positive controls urothelial carcinoma for GATA3, malignant melanoma for SOX10, normal ovary and thyroid for PAX8, normal breast parenchyma for ER and PR, and tonsil for Ki-67 labeling index - to validate the staining method. A negative control was prepared by substituting non-immune serum for the primary antibody, which resulted in no detectable staining. The expression status of ER, PR, HER2, and Ki-67 was evaluated according to the American Society of Clinical Oncology/College of American Pathologists guidelines (14-15). Fluorescence in situ hybridization was performed using ZytoLight SPEC HER2/CEN 17 dual-color probe (Zytovision, Bremerhaven, Germany) and observed under a fluorescence microscope at $1000 \mathrm{X}$ magnification to confirm the equivocal staining pattern of HER2. The Ki-67 labeling index was supplemented with HiPath ProTM computer-aided immunohistochemistry analysis (GenASIs, Carlsbad, CA, USA).

Interpretation of GATA3, SOX10, and PAX8 expression. Assessment of GATA3, SOX10, and PAX8 expression was based on the intensity (weak, intermediate, or strong) of nuclear staining and the proportion of stained carcinoma cells $(0 \%-100 \%)$, as previously described (10) (Figure 1). GATA3 and SOX10 expression were scored as any degree of nuclear staining, and recorded as percentage $(0-100 \%)$ and intensity staining (weak, moderate, strong) (2). Any nuclear labeling $>1 \%$ of cells rendered the sample positive (1). Tumors expressing PAX8 in $>10 \%$ of the cells with moderate to strong intensity of nuclear expression were considered PAX8-positive tumors. All IHC slides were independently reviewed by three pathologists (KN, SWK, and SID) for consensus. 

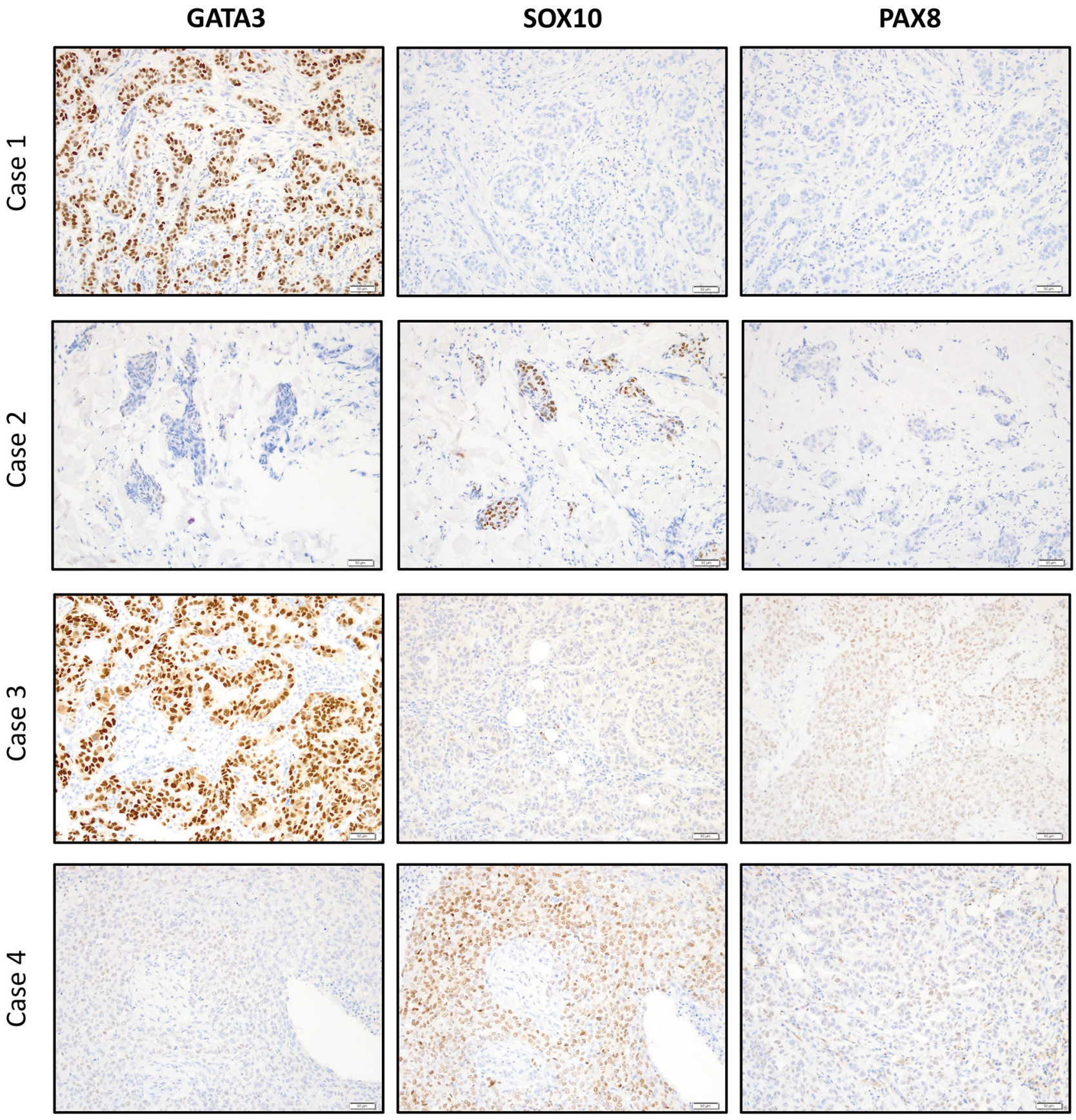

Figure 1. Histology. Representative images of immunohistochemical staining of GATA-binding protein 3 (GATA3), Sry-related HMG box 10 (SOX10), and paired box 8 (PAX8)-positive metastatic breast carcinoma (MBC). In case 1 (luminal A subtype type), tumor cell nuclei are diffusely positive for GATA3, and negative for SOX10 and PAX8. In contrast, in case 2 (triple-negative breast cancer), tumor cells are negative for GATA3 and PAX8, and positive for SOX10. In cases 3 and 4 , either GATA3 or SOX10 are weakly positive, and PAX8 shows patchy expression with weak to moderate intensity. Original magnification: A-F, 200x.

Statistical analysis. A chi-square test or Fisher's exact test was used to determine an association between categorical variables. An independent $t$-test was used to compare the means between the two continuous variables. Statistical analyses were performed using PASW Statistics for Windows (version 28.0; IBM SPSS, Armonk, NY, USA). Statistical significance was set at $p<0.05$. 


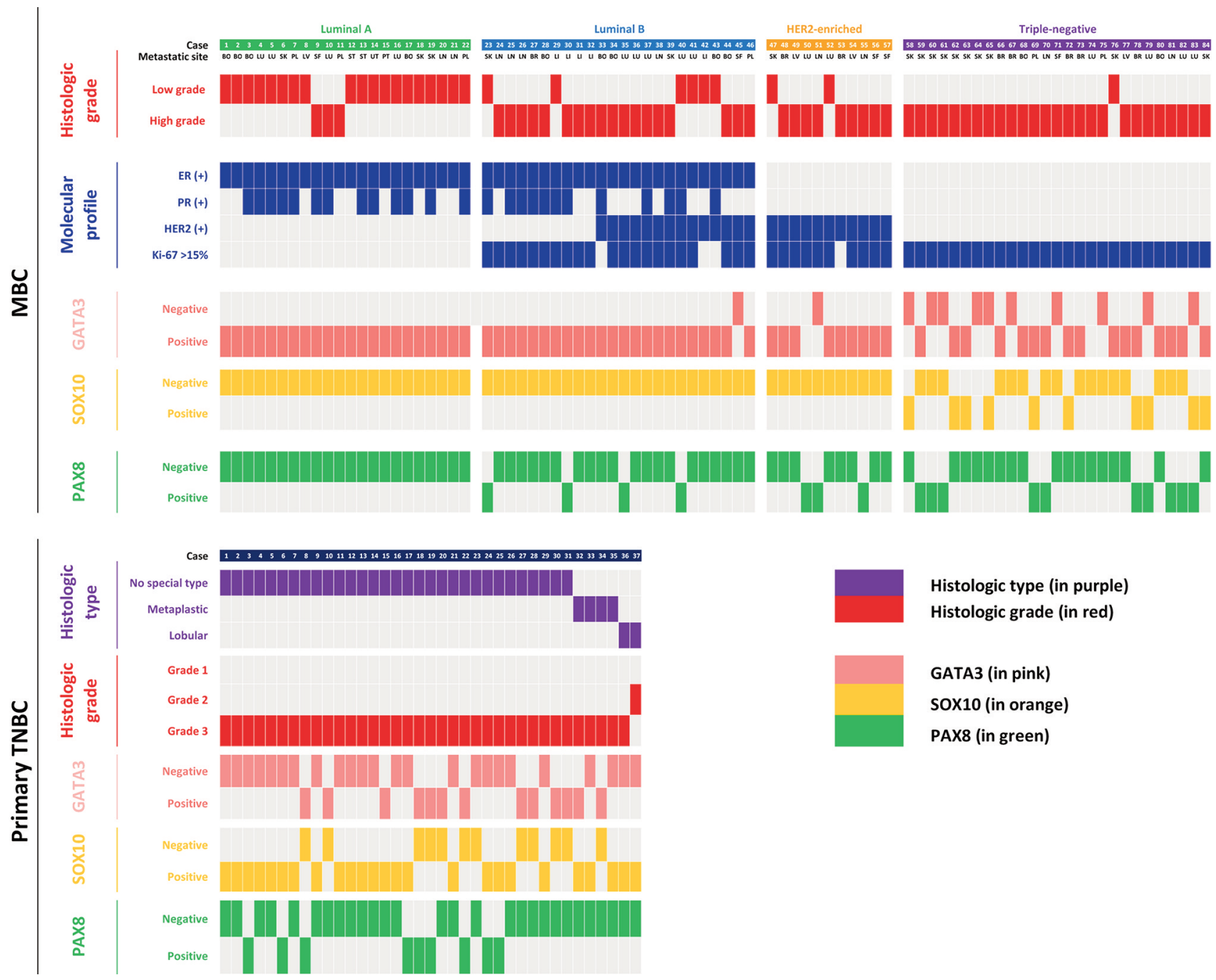

Figure 2. Histopathological features, molecular profiles, and immunohistochemical markers (GATA3, SOX10, and PAX8) expression status in metastatic breast cancer $(M B C)$ and primary triple-negative breast cancer (TNBC) of 121 patients. ER: Estrogen receptor; PR: progesterone receptor; HER2: human epidermal growth factor receptor; NST: no special type; GATA3: GATA-binding protein 3; SOX10: Sry-related HMG box 10; PAX8: Paired box 8.

\section{Results}

Patient characteristics. Figure 2 illustrates the histopathological features, molecular subtypes, and GATA3, SOX10, and PAX8 expression status in MBCs from 84 patients and p-TNBC from 37 patients. The mean age at the time of diagnosis of MBC and p-TNBC was 56.6 years (range=34-92 years) and 54.4 years (range=35-81 years), respectively. The metastatic sites identified were: i) skin $(\mathrm{n}=16)$, ii) lung $(\mathrm{n}=15)$, iii) bone $(\mathrm{n}=11)$, iv) liver $(\mathrm{n}=9), \mathrm{v})$ brain $(n=8)$, vi) pleura $(n=6)$, vii) lymph nodes [mediastinal $(n=4)$, cervical $(n=4)$, and supraclavicular $(n=2)]$, viii) soft tissue $(n=5)$, ix) stomach $(n=2), x)$ uterus $(n=1)$, and $x i)$ peritoneum $(n=1)$. The molecular subtypes of the $84 \mathrm{MBCs}$ were: i) TNBC ( $n=27)$, ii) luminal $B(n=24)$, iii) luminal A $(n=22)$, and iv) HER2-enriched $(n=11)$. The histopathological subtypes of the p-TNBCs were: i) invasive breast carcinoma of no special type (IBC-NST; $n=31$ ), ii) metaplastic carcinoma $(n=4)$, iii) invasive lobular carcinoma (ILC; $n=1)$, and iv) pleomorphic ILC ( $n=1)$. We subdivided MBC grades into a two-tier system (low-grade $v s$. high-grade), based on the predominant growth patterns and nuclear atypia. Using this grading method, 28 cases $(34 \%)$ of MBC were categorized as low-grade. The molecular subtypes of 28 lowgrade MBCs were: i) luminal A ( $n=19)$, ii) luminal $B(n=6,4$ HER2 positive cases), iii) HER2-enriched $(n=2)$, and iv) TNBC $(n=1)$. The Nottingham grades for the $37 \mathrm{p}$-TNBCs were grade $2(n=36)$ and grade $3(n=1)$. The molecular 
Table I. Statistical analysis of PAX8 expression status in metastatic breast carcinoma.

\begin{tabular}{|c|c|c|c|c|c|c|c|c|c|c|}
\hline \multirow[b]{2}{*}{ Parameters } & \multirow[b]{2}{*}{ Case } & \multicolumn{2}{|c|}{ GATA3 } & \multirow[b]{2}{*}{$p$-Value } & \multicolumn{2}{|c|}{ SOX-10 } & \multirow[b]{2}{*}{$p$-Value } & \multicolumn{2}{|c|}{ PAX8 } & \multirow[b]{2}{*}{$p$-Value } \\
\hline & & $\begin{array}{c}\text { Negative } \\
(\mathrm{n}=14) \\
\mathrm{n}(\%)\end{array}$ & $\begin{array}{c}\text { Positive } \\
(\mathrm{n}=70) \\
\mathrm{n}(\%)\end{array}$ & & $\begin{array}{c}\text { Negative } \\
(\mathrm{n}=74) \\
\mathrm{n}(\%)\end{array}$ & $\begin{array}{c}\text { Positive } \\
(\mathrm{n}=10) \\
\mathrm{n}(\%)\end{array}$ & & $\begin{array}{c}\text { Negative } \\
(\mathrm{n}=66) \\
\mathrm{n}(\%)\end{array}$ & $\begin{array}{c}\text { Positive } \\
(\mathrm{n}=18) \\
\mathrm{n}(\%)\end{array}$ & \\
\hline Age (mean years) & & 56.3 & 55.4 & 0.134 & 55.6 & 57.5 & 0.334 & 57.3 & 53.4 & 0.216 \\
\hline Histological grade & & & & 0.029 & & & 0.027 & & & 0.003 \\
\hline Low grade & $(\mathrm{n}=28)$ & $1(7)$ & 27 (39) & & $28(38)$ & $0(0)$ & & $26(39)$ & $2(11)$ & \\
\hline High grade & $(\mathrm{n}=55)$ & $13(93)$ & $43(61)$ & & $46(62)$ & $10(100)$ & & $40(51)$ & $16(89)$ & \\
\hline ER & & & & 0.001 & & & $<0.001$ & & & 0.003 \\
\hline Negative & $(\mathrm{n}=37)$ & $12(86)$ & $1(37)$ & & $28(38)$ & $10(100)$ & & $24(36)$ & $14(77)$ & \\
\hline Positive & $(n=46)$ & $2(14)$ & $44(63)$ & & $46(62)$ & $0(0)$ & & $42(66)$ & $4(25)$ & \\
\hline PR & & & & 0.055 & & & 0.058 & & & 0.247 \\
\hline Negative & $(\mathrm{n}=65)$ & $13(93)$ & $1(66)$ & & $49(66)$ & $10(100)$ & & $44(67)$ & $15(83)$ & \\
\hline Positive & $(n=18)$ & $1(7)$ & $24(34)$ & & $25(34)$ & $0(0)$ & & $22(33)$ & $3(17)$ & \\
\hline HER2 & & & & 0.331 & & & 0.055 & & & 0.558 \\
\hline Negative & $(n=59)$ & $12(86)$ & $49(70)$ & & $51(69)$ & $10(100)$ & & $49(74)$ & $12(67)$ & \\
\hline Positive & $(n=24)$ & $2(13)$ & $21(30)$ & & $23(31)$ & $0(0)$ & & $17(26)$ & $6(33)$ & \\
\hline Molecular subtype & & & & 0.001 & & & $<0.001$ & & & 0.008 \\
\hline Luminal A & $(n=22)$ & $1(4)$ & $21(96)$ & $0.101 *$ & $22(100)$ & $0(0)$ & $0.057 *$ & $22(100)$ & $0(0)$ & $0.008^{*}$ \\
\hline Luminal B & $(n=24)$ & $1(4)$ & $23(96)$ & $0.101 * *$ & $24(102)$ & $0(0)$ & $0.056 * *$ & $20(83)$ & $4(16)$ & $0.765 * *$ \\
\hline HER2-enriched & $(n=11)$ & $1(9)$ & $10(91)$ & $0.681 * * *$ & $11(100)$ & $0(0)$ & $0.345^{* * *}$ & $7(60)$ & $4(40)$ & $0.106 * * *$ \\
\hline Triple-negative & $(n=27)$ & $11(41)$ & $16(60)$ & $<0.001 * * * *$ & $17(63)$ & $10(37)$ & $<0.001 * * * *$ & $17(66)$ & $10(33)$ & $0.053 * * * *$ \\
\hline
\end{tabular}

*Luminal A versus others; **Luminal B versus others; ***HER2-enriched versus others; ****Triple-negative versus others. GATA3: GATA-binding protein 3; SOX10: Sry-related HMG box 10; PAX8: Paired box 8; ER: estrogen receptor; PR: progesterone receptor; HER2: human epidermal growth factor receptor. Bold values indicate statistical significance $(p<0.05)$.

subtypes of the 55 high-grade MBCs were: i) luminal A ( $\mathrm{n}=3)$, ii) luminal $\mathrm{B}(\mathrm{n}=18,14$ HER2 positive cases), iii) HER2-enriched $(n=9)$, and iv) triple-negative $(n=27)$.

Marker expression patterns in breast cancer. When scoring for nuclear staining for GATA3, this was found to be in 5\%$100 \%$ of tumor cells. Most of the 70 GATA3-positive tumors $(n=64 / 70,91 \%)$ showed at least $40 \%$ of tumor cells with moderate or strong nuclear staining (Figure 1, case 1). In SOX10-positive cases, most samples showed diffuse and strong nuclear positivity $(\mathrm{n}=7 / 10,70 \%)$ (Figure 1 , case 2 ). In PAX8-positive cases, $18(\mathrm{n}=18 / 84,11 \%)$ displayed a distinct nuclear PAX8 expression with more than $10 \%$ of the tumor cells stained (Figure 1, cases 3 and 4).

Interestingly, staining patterns of all the aforementioned three markers were significantly different in TNBC compared to other molecular subtypes. For GATA3, six cases $(n=6 / 70$, $8 \%$ ) showed weak nuclear staining in $<40 \%$ of cells, all of which were TNBC (Figure 1, case 3). For SOX10, all cases were strongly positive in $40 \%-70 \%$ of tumor cells, except for three cases, which showed a moderate nuclear positivity (Figure 1, case 4). Likewise, strong and diffuse PAX8positive cases were dominant in TNBC $(n=6 / 7,86 \%)$.
Association between breast markers expression and clinicopathologic parameters in $M B C$. The association between the expression pattern of the breast markers status and various pathological parameters in MBCs are summarized in Table I. Positive expression of all three markers was significantly associated with high-grade features (GATA3, $p=0.029$; SOX10, $p=0.027$; PAX8, $p=0.003$ ), and ER negativity (non-luminal; GATA3, $p=0.001 ; \mathrm{SOX} 10, p<0.001 ; \mathrm{PAX} 8, p=0.003)$. Of the four molecular subtypes of MBC, GATA3 was significantly expressed in non-TNBC cases $[\mathrm{n}=54 / 70,77 \%, p<0.001 ; \mathrm{i})$ luminal A, $96 \%$ (21/22); ii) luminal B, $96 \%$ (23/24); iii) HER2-enriched, 91\% (1/11); iv) TNBC, 60\% (16/27)]. Of these cases, only $3(6 \%)$ cases of non-TNBC were GATA3negative. Conversely, all 10 SOX10-positive cases were TNBC. None of the other molecular subtypes were SOX10positive $(p<0.001)$. The highest PAX8 expression was found in the HER2-enriched subtype (4/10, 40\%), followed by the triple-negative $(10 / 27,33 \%)$ and luminal B subtypes $(4 / 24,16 \%)$. The luminal A subtype was PAX8-negative $(0 / 22,0 \%)$. PAX8 expression was significantly higher in the non-luminal A subtype compared to the luminal A subtype $(p=0.004)$. 
Table II. Combined expression of SOX10, GATA3, and PAX8 in breast cancer.

\begin{tabular}{|c|c|c|c|c|c|c|c|c|c|c|c|c|}
\hline & \multicolumn{3}{|c|}{$\begin{array}{l}\text { Metastatic breast } \\
\text { cancer }(\mathrm{n}=84)\end{array}$} & \multicolumn{3}{|c|}{$\begin{array}{c}\text { Metastatic } \\
\text { non-TNBC }(\mathrm{n}=57)\end{array}$} & \multicolumn{3}{|c|}{$\begin{array}{c}\text { Metastatic } \\
\text { TNBC }(n=27)\end{array}$} & \multicolumn{3}{|c|}{$\begin{array}{c}\text { Primary } \\
\text { TNBC }(n=37)\end{array}$} \\
\hline & + & - & Sensitivity & + & - & Sensitivity & + & - & Sensitivity & + & - & Sensitivity \\
\hline GATA3+ & 70 & 14 & $0.83(70 / 84)$ & 54 & 3 & $0.95(54 / 57)$ & 16 & 11 & $0.37(16 / 27)$ & 14 & 23 & $0.38(14 / 37)$ \\
\hline SOX10+ & 10 & 74 & $0.12(10 / 84)$ & 0 & 57 & $0(0 / 57)$ & 11 & 16 & $0.41(11 / 27)$ & 23 & 14 & $0.64(23 / 37)$ \\
\hline PAX8- & 18 & 66 & $0.79(66 / 84)$ & 8 & 49 & $0.86(49 / 57)$ & 10 & 17 & $0.63(17 / 27)$ & 22 & 15 & $0.60(15 / 37)$ \\
\hline GATA3+ or SOX10+ & 75 & 9 & $0.89(74 / 84)$ & 54 & 3 & $0.95(54 / 57)$ & 21 & 6 & $0.78(21 / 27)$ & 29 & 8 & $0.78(29 / 37)$ \\
\hline SOX10+ or PAX8- & 70 & 14 & $0.83(70 / 84)$ & 49 & 8 & $0.86(49 / 57)$ & 21 & 6 & $0.78(21 / 27)$ & 30 & 7 & $0.80(30 / 37)$ \\
\hline GATA3+ or PAX8- & 79 & 5 & $0.94(79 / 84)$ & 55 & 2 & $0.95(55 / 57)$ & 24 & 3 & $0.89(24 / 27)$ & 33 & 4 & $0.90(33 / 37)$ \\
\hline $\begin{array}{l}\text { GATA3+ or SOX10+ } \\
\text { or PAX8- }\end{array}$ & 78 & 6 & $0.94(78 / 84)$ & 55 & 2 & $0.95(55 / 57)$ & 23 & 4 & $0.93(23 / 27)$ & 35 & 2 & $0.94(35 / 37)$ \\
\hline
\end{tabular}

TNBC: Triple-negative breast cancer; GATA3: GATA-binding protein 3; SOX10: Sry-related HMG box 10; PAX8: Paired box 8.

Detection rate of single markers in breast cancer. Table II shows the sensitivity of all the three markers in $\mathrm{MBC}$, metastatic non-TNBC (m-nonTNBC), metastatic TNBC (mTNBC), and p-TNBC. At least either GATA3 or SOX-10 were positive in all TNBC metastases. Overall, MBC showed a high GATA3-positive rate (Table II; $n=70 / 84,83 \%$ ), but low SOX10- and PAX8-positive rates $(n=10 / 84,12 \%$ and $\mathrm{n}=18 / 84$, $11 \%$, respectively). GATA3 had the highest sensitivity towards m-nonTNBC (0.95) and lowest sensitivity towards m-TNBC (0.37). Conversely, SOX10 had the lowest overall sensitivity $(0.12)$, but in $\mathrm{m}$-TNBC it had a high sensitivity, even when compared to GATA3 (0.59 vs. 0.37 , respectively). Of the 14 GATA3-negative cases, 4 that were positive for SOX10 were all m-TNBC cases. Most SOX10negative cases $(n=64 / 70,91 \%)$ were GATA3-positive and mnonTNBC $(n=54 / 64,84 \%)$. Nearly all MBC cases $(n=74 / 84$, $88 \%$ ) were positive for either GATA3 or SOX10, with $8 \%$ $(n=6 / 74)$ of them being positive for both markers and 10 cases (13.5\%) being negative for both (Figure 2). More SOX10-positive tumors were observed in GATA3-negative MBC ( $\mathrm{n}=4 / 14,30 \%) v s$. GATA3-positive MBC $(\mathrm{n}=6 / 64,9 \%)$ $(p=0.05)$. Notably, it seemed that GATA3 and SOX10 were expressed in different subgroups of MBC, and GATA3 expression was negatively correlated with SOX10 (Pearson correlation coefficient $=-0.23, p=0.03$ ).

PAX8 expression is commonly negative in breast cancers, but is aberrantly seen in TNBC (13). Therefore, we observed negative levels of PAX8 in tumors separately from other markers to rule out metastases in other tumors. Similar to GATA3, PAX8 displayed a lower sensitivity in m-TNBC (0.63) compared to m-nonTNBC (0.86). PAX8 expression was observed in $13(19 \%)$ of the 70 GATA3-positive MBCs, and in $5(36 \%)$ of the GATA3-negative MBC cases, indicating that PAX8 expression was fairly higher in GATA3-negative compared to the GATA3-positive MBC $(p=0.03)$. Four out of $10 \mathrm{SOX} 10$-positive MBC cases were
PAX8-positive and TNBCs. Four of the six GATA3 and SOX10 dual negative m-TNBC cases were PAX8-negative.

Combination of GATA3, SOX10, and PAX8 markers in breast cancer. We evaluated the diagnostic ability of each marker combination. In a single marker study, SOX10 had a high sensitivity in TNBC and a negative association with GATA3 in breast cancer subtypes. When GATA3 and SOX10 were combined, the detection rate improved compared to that for either of the markers expressed on their own (Table II). The overall sensitivity for MBC was 0.88 for GATA3/SOX10 vs. 0.83 for GATA3 alone and 0.12 for SOX10 alone. For GATA3, which on its own showed a low sensitivity in m-TNBC $(0.37)$, the detection rate increased when it was present together with SOX10 (0.78). Coexpression of GATA3 and SOX10 with PAX8 showed the highest detection rate (MBC, 0.94; m-nonTNBC, 0.95 ; mTNBC, 0.93).

In $\mathrm{MBC}$, we observed that the staining patterns and expression status were more diverse in m-TNBC subtypes compared to the m-nonTNBC subtypes. Next, we investigated these markers in $37 \mathrm{p}$-TNBC cases and analyzed the detection rate (Table II). Again, statistical analysis showed a significant relationship between GATA3, SOX10, and PAX8 expression and the clinicopathological parameters in the $37 \mathrm{p}$-TNBCs. The positive rate of Sox10 expression in $\mathrm{p}$ - and $\mathrm{m}$-TNBC was significantly higher than that of the luminal $\mathrm{A}$ and $\mathrm{B}$, and HER2-enriched subtypes (both $p<0.001$ ), as shown in Table I.

\section{Discussion}

In this study, we evaluated the diagnostic value of GATA3, SOX10, and PAX8 in $84 \mathrm{MBC}$ cases and demonstrated that combining these markers in histology helps to differentiate $\mathrm{MBC}$ from other malignancies. The diagnosis of MBC and 
its differentiation from other anatomical sites is a common necessity in pathology practice. Most commonly diagnosed MBCs are metastatic, high-grade tumors of the axillary or cervical lymph nodes in female patients (4). The combinatory profiling of cytokeratins (CK) 7 and 20 expression is a classic and useful method to identify the origin of a carcinoma of unknown primary origin (12). Female patients with a $\mathrm{CK} 7+\mathrm{CK} 20$ - profile usually have the organs above the diaphragm (lung, breast, thyroid, and salivary gland) as well as the gynecologic tract (uterus and ovary) affected (13). The use of organ-specific markers, such as the thyroid transcription factor-1 (TTF-1) or hormone receptor markers, including ER and $\mathrm{PR}$, in conjunction with GATA3/SOX10/PAX8 may help to further classify the tumor origin. The absence or low levels of GATA3 and SOX10 expression in lung cancer is particularly relevant, as metastatic lung adenocarcinoma is one of the most common sites for MBC (13). Positivity for GATA3 or SOX10 and negativity for TTF-1 favor the mammary origin and vice versa (14). Since the expression of ER and PR is similar in both breast and gynecological cancers, especially nonTNBC, their accurate diagnosis might prove difficult. In this setting, PAX8 positivity is a more prominent feature in gynecological cancer compared to breast cancer. Notably, in breast cancer, especially TNBC, the aberrant expression of PAX8 and the absence of hormone receptor expression may affect the accuracy of the diagnosis (14). In our study, there were two cases where the expression of GATA3 and SOX10 in TNBC was accompanied by aberrant expression of PAX8 However, both these cases showed weakly positive results. Therefore, when a weak PAX8-positive result is observed in GATA3- and SOX10-negative tumors, the possibility of MBC should also be considered.

We observed that GATA3 is a sensitive lineage marker for breast cancer, which is consistent with previous studies (2). GATA3 is considered the most sensitive marker for breast cancer compared to other standard breast lineage markers, such as gross cystic disease fluid protein 15 (GCDFP15) and MMG (5), even though together with other standard markers it has a low sensitivity in TNBC (2). In our study, GATA3 was still less sensitive in TNBC compared to non-TNBC, which could be due to its involvement in the ER signaling pathway (15). A major drawback of GATA3 as a mammary lineage marker is that its sensitivity is highly correlated with the ER status (1). In ER-positive breast cancers, GATA3positivity is between $91 \%$ and $100 \%$, but the sensitivity is relatively low in TNBC, supporting our results. Therefore, GATA3 should be supplemented with additional complementary markers like SOX10, which is expressed only in TNBC (1). In our study, the combination of GATA3/SOX10 demonstrated a high overall detection rate $(88 \%)$ for MBC. Importantly, this GATA3/SOX10 combination increased the detection rate of TNBC from approximately $30 \%$ (using a single marker) to $74 \%$, as SOX10 identified many GATA3-negative TNBCs. This is a significant finding, as TNBCs lack hormone receptor expression, making it hard to identify the breast as a potential primary site in routine practice.

Another challenge is to differentiate metastatic breast cancer from other ER-positive cancers of Müllerian origin (16). For example, the differential diagnosis of ovarian highgrade serous carcinoma and high-grade breast carcinoma at metastatic sites based on histological features alone can be challenging (3). Therefore, the diagnosis is often supplemented by several immunohistochemical markers to establish the origin of the carcinoma (17). Testing for PAX8 expression may help differentiate between MBC, especially non-TNBC, and cancers of Müllerian origin. PAX8 is a sensitive and specific marker for carcinomas of the thyroid, kidney, and Müllerian organs but is not expressed in breast carcinoma (18). Since GATA3 is a diagnostic surrogate marker of breast carcinoma, GATA3 expression and the absence of PAX8 expression aid in the diagnostic separation between mammary and Müller origin cancer types (19). In our study, the combination of GATA3/SOX10/PAX8 demonstrated a high overall detection rate $(94 \%)$ for $\mathrm{MBC}$ (94\%), consistent with a previous study (19).

Meanwhile, metastatic malignant melanoma can simulate a variety of high-grade tumors, including TNBC, suggesting that SOX10 expression may not help differentiate TNBC from malignant melanoma (20). Therefore, SOX10 expression levels in such scenarios should be interpreted in conjunction with other melanocyte markers, namely Human Melanoma Black (HMB) 45 and Melan-A, to avoid diagnostic pitfalls (7). SOX10 may suggest the primary origin to be the mammary gland if the metastatic lesion is TNBC, and the use of appropriate diagnostic tools, such as IHC results of positive cytokeratin, exclude melanoma and indicate MBC. Primary salivary gland tumors may also express SOX10 and/or GATA3 (21-24) complicating matters even more. Interestingly, this complication reflects the structural similarity between the salivary glands and breast, both of which play an exocrine role. Salivary gland tumors have similar morphology, immunohistochemical profile, and molecular features as breast cancers (22). Therefore, the SOX10/GATA3 marker combination is not useful for rare breast/salivary gland tumors, where cancer spreads to the breast/salivary or cervical lymph nodes (24-26). Careful clinical or radiological evaluations are crucial for accurate diagnosis.

In summary, we proposed combinations of IHC markers based on the molecular profiles of the MCB subtypes for their differential diagnosis. The combination of GATA3, SOX10, and PAX8 increased the detection rate of MBC, particularly of TNBC. This combination can be used to identify MBCs that exhibit various morphological and immunological profiles. 


\section{Conflicts of Interest}

The Authors declare no conflicts of interest regarding this study.

\section{Authors' Contributions}

SWK, HYW, SID, and KN made substantial contributions to the conception and design of the study, acquisition of data, analysis and interpretation of the data, as well as drafting the manuscript and revising it critically for important intellectual content, and providing final approval of the version to be published.

\section{Acknowledgements}

This research was supported by the Basic Science Research Program through the National Research Foundation of Korea (NRF), funded by the Ministry of Science, ICT, and Future Planning (NRF-2020R1G1A1003692).

\section{References}

1 Tozbikian GH and Zynger DL: A combination of GATA3 and SOX10 is useful for the diagnosis of metastatic triple-negative breast cancer. Hum Pathol 85: 221-227, 2019. PMID: 30468800. DOI: 10.1016/j.humpath.2018.11.005

2 Aphivatanasiri C, Li J, Chan R, Jamidi SK, Tsang JY, Poon IK, Shao Y, Tong J, To KF, Chan SK, Tam F, Cheung SY, Shea KH and Tse GM: Combined SOX10 GATA3 is most sensitive in detecting primary and metastatic breast cancers: a comparative study of breast markers in multiple tumors. Breast Cancer Res Treat 184(1): 11-21, 2020. PMID: 32737715. DOI: 10.1007/ s10549-020-05818-9

3 Van Poznak C, Somerfield MR, Bast RC, Cristofanilli M, Goetz MP, Gonzalez-Angulo AM, Hicks DG, Hill EG, Liu MC, Lucas W, Mayer IA, Mennel RG, Symmans WF, Hayes DF and Harris LN: Use of biomarkers to guide decisions on systemic therapy for women with metastatic breast cancer: American Society of Clinical Oncology clinical practice guideline. J Clin Oncol 33(24): 26952704, 2015. PMID: 26195705. DOI: 10.1200/JCO.2015.61.1459

4 Cimino-Mathews A: Novel uses of immunohistochemistry in breast pathology: interpretation and pitfalls. Mod Pathol 34(Suppl 1): 62-77, 2021. PMID: 33110239. DOI: 10.1038/ s41379-020-00697-3

5 Wendroth SM, Mentrikoski MJ and Wick MR: GATA3 expression in morphologic subtypes of breast carcinoma: a comparison with gross cystic disease fluid protein 15 and mammaglobin. Ann Diagn Pathol 19(1): 6-9, 2015. PMID: 25544392. DOI: 10.1016/j.anndiagpath.2014.12.001

6 Lewis GH, Subhawong AP, Nassar H, Vang R, Illei PB, Park BH and Argani P: Relationship between molecular subtype of invasive breast carcinoma and expression of gross cystic disease fluid protein 15 and mammaglobin. Am J Clin Pathol 135(4): 587-591, 2011. PMID: 21411781. DOI: 10.1309/ AJCPMFR6OA8ICHNH

7 Nelson ER, Sharma R, Argani P and Cimino-Mathews A: Utility of Sox 10 labeling in metastatic breast carcinomas. Hum Pathol 67: 205-210, 2017. PMID: 28843711. DOI: 10.1016/j.humpath. 2017.08.011
8 Yang Y, Lu S, Zeng W, Xie S and Xiao S: GATA3 expression in clinically useful groups of breast carcinoma: a comparison with GCDFP15 and mammaglobin for identifying paired primary and metastatic tumors. Ann Diagn Pathol 26: 1-5, 2017. PMID: 28038704. DOI: 10.1016/j.anndiagpath.2016.09.011

9 Sejben A, Vörös A, Golan A, Zombori T and Cserni G: The added value of SOX10 immunohistochemistry to other breast markers in identifying cytokeratin 5-positive triple negative breast cancers as of mammary origin. Pathobiology 88(3): 228233, 2021. PMID: 33567441. DOI: 10.1159/000512006

10 Chiu K, Ionescu DN and Hayes M: SOX10 expression in mammary invasive ductal carcinomas and benign breast tissue. Virchows Arch 474(6): 667-672, 2019. PMID: 30903273. DOI: 10.1007/s00428-019-02557-1

11 Kriegsmann K, Flechtenmacher C, Heil J, Kriegsmann J, Mechtersheimer G, Aulmann S, Weichert W, Sinn HP and Kriegsmann M: Immunohistological expression of SOX-10 in triple-negative breast cancer: A descriptive analysis of 113 samples. Int J Mol Sci 21(17): 6407, 2020. PMID: 32899175. DOI: $10.3390 /$ ijms21176407

12 Min YS, Yi EH, Lee JK, Choi JW, Sim JH, Kang JS, Kim YN, Juhnn YS, Kim HR and Ye SK: CK20 expression enhances the invasiveness of tamoxifen-resistant MCF-7 cells. Anticancer Res 32(4): 1221-1228, 2012. PMID: 22493352.

13 Tornos C, Soslow R, Chen S, Akram M, Hummer AJ, AbuRustum N, Norton L and Tan LK: Expression of WT1, CA 125 , and GCDFP-15 as useful markers in the differential diagnosis of primary ovarian carcinomas versus metastatic breast cancer to the ovary. Am J Surg Pathol 29(11): 14821489, 2005. PMID: 16224215. DOI: 10.1097/01.pas. 0000176429.88702 .36

$14 \mathrm{Kim}$ SW, Kim HS and Na K: Characterization of paired box 8 (PAX8)-expressing metastatic breast carcinoma. Anticancer Res 40(10): 5925-5932, 2020. PMID: 32988924. DOI: $10.21873 /$ anticanres. 14613

15 Nonaka D, Chiriboga L and Soslow RA: Expression of pax8 as a useful marker in distinguishing ovarian carcinomas from mammary carcinomas. Am J Surg Pathol 32(10): 1566-1571, 2008. PMID: 18724243. DOI: 10.1097/PAS.0b013e31816d71ad

16 Asch-Kendrick R and Cimino-Mathews A: The role of GATA3 in breast carcinomas: a review. Hum Pathol 48: 37-47, 2016. PMID: 26772397. DOI: 10.1016/j.humpath.2015.09.035

17 Conner JR and Hornick JL: Metastatic carcinoma of unknown primary: diagnostic approach using immunohistochemistry. Adv Anat Pathol 22(3): 149-167, 2015. PMID: 25844674. DOI: 10.1097/PAP.0000000000000069

18 Domfeh AB, Carley AL, Striebel JM, Karabakhtsian RG, Florea $\mathrm{AV}$, McManus $\mathrm{K}$, Beriwal $\mathrm{S}$ and Bhargava R: WT1 immunoreactivity in breast carcinoma: selective expression in pure and mixed mucinous subtypes. Mod Pathol 21(10): 12171223, 2008. PMID: 18469795. DOI: 10.1038/modpathol.2008.69

19 Kilgore MR, Bosch DE, Adamson KH, Swanson PE, Dintzis SM and Rendi MH: Unexpected PAX8 immunoreactivity in metastatic high-grade breast cancer. Appl Immunohistochem Mol Morphol 27(9): 637-643, 2019. PMID: 30358609. DOI: 10.1097/PAI.0000000000000707

20 Statz E and Jorns JM: Cytokeratin 7, GATA3, and SOX-10 is a comprehensive panel in diagnosing triple negative breast cancer brain metastases. Int J Surg Pathol 29(5): 470-474, 2021. PMID: 33543662. DOI: $10.1177 / 1066896921990717$ 
21 Harbhajanka A, Chahar S, Miskimen K, Silverman P, Harris L, Williams N, Varadan V and Gilmore H: Clinicopathological, immunohistochemical and molecular correlation of neural crest transcription factor SOX10 expression in triple-negative breast carcinoma. Hum Pathol 80: 163-169, 2018. PMID: 29894722. DOI: $10.1016 /$ j.humpath.2018.06.007

22 Miettinen M, Fernandez M, Franssila K, Gatalica Z, Lasota J and Sarlomo-Rikala M: Microphthalmia transcription factor in the immunohistochemical diagnosis of metastatic melanoma: comparison with four other melanoma markers. Am J Surg Pathol 25(2): 205-211, 2001. PMID: 11176069. DOI: 10.1097/ 00000478-200102000-00008

23 Miettinen M, McCue PA, Sarlomo-Rikala M, Biernat W, Czapiewski P, Kopczynski J, Thompson LD, Lasota J, Wang Z and Fetsch JF: Sox10 - a marker for not only schwannian and melanocytic neoplasms but also myoepithelial cell tumors of soft tissue: a systematic analysis of 5134 tumors. Am J Surg Pathol 39(6): 826-835, 2015. PMID: 25724000. DOI: 10.1097/PAS. 0000000000000398

24 Cimino-Mathews A, Subhawong AP, Elwood H, Warzecha HN, Sharma R, Park BH, Taube JM, Illei PB and Argani P: Neural crest transcription factor Sox10 is preferentially expressed in triple-negative and metaplastic breast carcinomas. Hum Pathol 44(6): 959-965, 2013. PMID: 23260325. DOI: 10.1016/ j.humpath.2012.09.005
25 Ohtomo R, Mori T, Shibata S, Tsuta K, Maeshima AM, Akazawa C, Watabe Y, Honda K, Yamada T, Yoshimoto S, Asai M, Okano $\mathrm{H}$, Kanai $\mathrm{Y}$ and Tsuda H: SOX10 is a novel marker of acinus and intercalated duct differentiation in salivary gland tumors: a clue to the histogenesis for tumor diagnosis. Mod Pathol 26(8): 1041-1050, 2013. PMID: 23558573. DOI: 10.1038/modpathol. 2013.54

26 Kim H, Gim JA, Kim CY and Kim A: Intratumor heterogeneity of synchronous in situ and invasive breast carcinoma revealed using multi-region exome sequencing. Anticancer Res 41(8): 3779-3787, 2021. PMID: 34281837. DOI: 10.21873/anticanres. 15170
Received August 11, 2021

Revised October 15, 2021

Accepted October 19, 2021 